\title{
Fixed point theorems in modular vector spaces
}

\author{
Afrah A. N. Abdou ${ }^{a}$, Mohamed A. Khamsi ${ }^{b, c, *}$ \\ ${ }^{a}$ Department of Mathematics, Faculty of Sciences, King Abdulaziz University, Jeddah 21593, Saudi Arabia. \\ ${ }^{b}$ Department of Mathematical Sciences, The University of Texas at El Paso, EI Paso, TX 79968, U.S.A. \\ ${ }^{c}$ Department of Mathematics \& Statistics, King Fahd University of Petroleum and Minerals, Dhahran 31261, Saudi Arabia.
}

Communicated by C. Vetro

\begin{abstract}
In this work, we initiate the metric fixed point theory in modular vector spaces under Nakano formulation. In particular, we establish an analogue to Banach contraction principle, Browder and Göhde fixed point theorems for nonexpansive mappings in the modular sense. Then we finish by proving a common fixed point result of a commutative family of nonexpansive mappings in the modular sense. (C)2017 All rights reserved.
\end{abstract}

Keywords: Best approximant, electrorheological fluids, fixed point, modular vector spaces, Nakano, nonexpansive, uniformly convex.

2010 MSC: 47H09, 46B20, 47H10.

\section{Introduction}

In most of the recent references on fixed point theory in modular vector spaces, there is a lack of answers to some fundamental questions. One of which is the importance of modular vector spaces. The concept of a modular finds its root in the work of Orlicz [21] published in 1931. In this publication, Orlicz introduced the vector space

$$
X=\left\{\left(x_{n}\right) \in \mathbb{R}^{\mathbb{N}}: \sum_{n=1}^{\infty}\left|\lambda x_{n}\right|^{n}<\infty \text { for some } \lambda>0\right\} .
$$

Questions about the geometry and topological properties of the vector space $X$ were asked. The formal definition of a modular that captured the essence of the definition of $X$ was done by Nakano [18, 20]. Note that the vector space $X$ was a precursor to what is known as a variable exponent space [5]. These spaces have seen a major development in recent years. A systematic study of their vector topological properties, like reflexivity, separability, duality and embeddings, was initiated in 1991 by Koväčik and Rákosník [13]. But one of the driving forces for the rapid development of the theory of variable exponent spaces has been

\footnotetext{
*Corresponding author

Email addresses: aabdou@kau.edu.sa (Afrah A. N. Abdou), mohamed@utep.edu (Mohamed A. Khamsi) 
the model of electrorheological fluids introduced by Rajagopal and Ružička $[22,23]$. This model leads naturally function spaces which involve variable exponents. Electrorheological fluids are an example of smart materials, whose development is one of the major task in space engineering.

In this work, we initiate the fundamental properties in the development of the metric fixed point theory in modular vector spaces. In particular, we investigate the geometric properties of the vector space $X$ described above. This investigation allowed us to discover some interesting properties not known before.

For the readers interested into the metric fixed point theory, we recommend the book by Khamsi and Kirk [9] and the recent book by Khamsi and Kozlowski [10].

\section{Notations and definitions}

Throughout this work, $X$ stands for a linear vector space on the field $\mathbb{R}$.

Definition 2.1 ([17, 18]). A function $\rho: X \rightarrow[0, \infty]$ is called modular if the following hold:

(1) $\rho(x)=0$ if and only if $x=0$;

(2) $\rho(\alpha x)=\rho(x)$, if $|\alpha|=1$;

(3) $\rho(\alpha x+(1-\alpha) y) \leqslant \rho(x)+\rho(y)$, for any $\alpha \in[0,1]$;

for any $x, y \in X$. If (3) is replaced by

$$
\rho(\alpha x+(1-\alpha) y) \leqslant \alpha \rho(x)+(1-\alpha) \rho(y)
$$

for any $\alpha \in[0,1]$, and $x, y \in X$, then $\rho$ is called a convex modular.

A modular function on $X$ will give birth to a modular space in a natural fashion.

Definition 2.2. Let $\rho$ be a convex modular defined on $X$. The set

$$
X_{\rho}=\left\{x \in X: \lim _{\alpha \rightarrow 0} \rho(\alpha x)=0\right\},
$$

is called a modular space. The Luxemburg norm $\|\cdot\|_{\rho}: X_{\rho} \rightarrow[0, \infty)$ is defined by

$$
\|x\|_{\rho}=\inf \left\{\alpha>0: \rho\left(\frac{x}{\alpha}\right) \leqslant 1\right\} .
$$

Throughout, we will assume that $\rho$ is left-continuous, i.e., $\lim _{\alpha \rightarrow 1-} \rho(\alpha x)=\rho(x)$, for any $x \in X_{\rho}$.

Example 2.3. Since the 1930s many prominent mathematicians like Orlicz and Birnbaum recognized that using the methods of $\mathrm{L}^{\mathrm{p}}$-spaces alone created many complications and in some cases did not allow to solve some non-power type integral equations; see [2]. They considered spaces of functions with some growth properties different from the power type growth control provided by the $\mathrm{L}^{\mathrm{p}}$-norms. Orlicz and Birnbaum considered for instance function spaces defined as follows:

$$
\mathrm{L}^{\varphi}=\left\{\mathrm{f}: \mathbb{R} \rightarrow \mathbb{R}: \text { there exists } \lambda>0 \text { such that } \int_{\mathbb{R}} \varphi(\lambda|\mathrm{f}(\mathrm{x})|) \mathrm{dm}(\mathrm{x})<\infty\right\},
$$

where $\varphi:[0, \infty) \rightarrow[0, \infty)$ was assumed to be a convex function increasing to infinity, that is, the function which to some extent behaves similarly to power functions $\varphi(t)=t^{p}$. Let us mention two typical examples of such functions: $\varphi_{1}(t)=e^{t}-t-1$ or $\varphi_{2}(t)=e^{t^{2}}-1$. The possibility of introducing the structure of a linear metric in $\mathrm{L}^{\varphi}$ as well as the interesting properties of these spaces, later named Orlicz spaces, and many applications to differential and integral equations with kernels of nonpower types were among the reasons for the development of the theory of Orlicz spaces, their applications and generalizations. Clearly the modular functional associated to $\mathrm{L}^{\varphi}$ is

$$
\rho(f)=\int_{\mathbb{R}} \varphi(|f(x)|) \mathrm{dm}(x) .
$$


Associated to a modular function, we introduce a kind of modular topology that mimic the classical metric topology.

Definition 2.4 ([12]). Let $\rho$ be a modular defined on a vector space $X$.

(a) We say that a sequence $\left\{x_{n}\right\} \subset X_{\rho}$ is $\rho$-convergent to $x \in X_{\rho}$ if and only if $\rho\left(x_{n}-x\right) \rightarrow 0$. Note that the $\rho$-limit is unique if it exists.

(b) A sequence $\left\{x_{n}\right\} \subset X_{\rho}$ is called $\rho$-Cauchy if $\rho\left(x_{n}-x_{m}\right) \rightarrow 0$ as $n, m \rightarrow \infty$.

(c) We say that $X_{\rho}$ is $\rho$-complete if and only if any $\rho$-Cauchy sequence in $X_{\rho}$ is $\rho$-convergent.

(d) A set $C \subset X_{\rho}$ is called $\rho$-closed if for any sequence of $\left\{x_{n}\right\} \subset C$ which $\rho$-converges to $x$ implies that $x \in C$.

(e) A set $C \subset X_{\rho}$ is called $\rho$-bounded if $\delta_{\rho}(C)=\sup \{\rho(x-y) ; x, y \in C\}<\infty$.

(f) A set $K \subset X_{\rho}$ is called $\rho$-compact if any sequence $\left\{x_{n}\right\}$ in $K$ has a subsequence which $\rho$-converges to a point in $\mathrm{K}$.

(g) $\rho$ is said to satisfy the Fatou property if $\rho(x-y) \leqslant \liminf _{n \rightarrow \infty} \rho\left(x-y_{n}\right)$ whenever $\left\{y_{n}\right\} \rho$-converges to $y$, for any $x, y, y_{n}$ in $x_{\rho}$.

Note that the Fatou property plays an important role when studying the geometric properties of the modular. For example, if $\rho$ satisfies the Fatou property then the $\rho$-balls are $\rho$-closed, where a $\rho$-ball is any subset

$$
B_{\rho}(x, r)=\left\{y \in X_{\rho}: \rho(x-y) \leqslant r\right\},
$$

for any $x \in X_{\rho}$ and $r \geqslant 0$. A very important property associated to a modular which plays a central role in the study of modular vector spaces is the $\Delta_{2}$-condition.

Definition 2.5. Let $\rho$ be a modular defined on a vector space $X$. We say that $\rho$ satisfies the $\Delta_{2}$-condition if there exists $K \geqslant 0$ such that

$$
\rho(2 x) \leqslant K \rho(x)
$$

for any $x \in X_{\rho}$. The smallest such constant $K$ will be denoted by $\omega(2)$ [6].

A nice discussion about the importance of the $\Delta_{2}$-condition and its variants may be found in [10, 14 , 17].

\section{Modular uniform convexity}

Throughout $X$ is a vector space and $\rho$ a convex modular function. As we have seen, $\rho$ induces a natural norm $\|\cdot\|_{\rho}$ in $X_{\rho}$. Some of the early questions that mathematicians dealt with is whether the normed vector space $\left(X_{\rho},\|\cdot\|_{\rho}\right)$ is uniformly convex. The answer came as of no surprise that $\rho$ must satisfy some good behavior. In fact, this problem was fully investigated in Orlicz function spaces [5, 17]. The modular uniform convexity was initiated and studied by Nakano [20].

Definition 3.1 ([10]). We define the following uniform convexity type properties of the modular $\rho$ :

(a) Let $r>0$ and $\varepsilon>0$. Define

$$
D_{1}(r, \varepsilon)=\left\{(x, y): x, y \in X_{\rho}, \rho(x) \leqslant r, \rho(y) \leqslant r, \rho(x-y) \geqslant \varepsilon r\right\} .
$$

If $D_{1}(r, \varepsilon) \neq \emptyset$, let

$$
\delta_{1}(r, \varepsilon)=\inf \left\{1-\frac{1}{r} \rho\left(\frac{x+y}{2}\right):(x, y) \in D_{1}(r, \varepsilon)\right\} .
$$

If $D_{1}(r, \varepsilon)=\emptyset$, we set $\delta_{1}(r, \varepsilon)=1$. We say that $\rho$ satisfies (UC1) if for every $r>0$ and $\varepsilon>0$, we have $\delta_{1}(r, \varepsilon)>0$. Note that for every $r>0, D_{1}(r, \varepsilon) \neq \emptyset$, for $\varepsilon>0$ small enough. 
(b) We say that $\rho$ satisfies (UUC1) if for every $s \geqslant 0$ and $\varepsilon>0$, there exists $\eta_{1}(s, \varepsilon)>0$ depending on $s$ and $\varepsilon$ such that

$$
\delta_{1}(r, \varepsilon)>\eta_{1}(s, \varepsilon)>0, \quad \text { for } r>s .
$$

(c) Let $r>0$ and $\varepsilon>0$. Define

$$
D_{2}(r, \varepsilon)=\left\{(x, y): x, y \in X_{\rho}, \rho(x) \leqslant r, \rho(y) \leqslant r, \rho\left(\frac{x-y}{2}\right) \geqslant \varepsilon r\right\} .
$$

If $\mathrm{D}_{2}(\mathrm{r}, \varepsilon) \neq \emptyset$, let

$$
\delta_{2}(r, \varepsilon)=\inf \left\{1-\frac{1}{r} \rho\left(\frac{x+y}{2}\right):(x, y) \in D_{2}(r, \varepsilon)\right\} .
$$

If $D_{2}(r, \varepsilon)=\emptyset$, we set $\delta_{2}(r, \varepsilon)=1$. We say that $\rho$ satisfies (UC2) if for every $r>0$ and $\varepsilon>0$, we have $\delta_{2}(r, \varepsilon)>0$. Note that for every $r>0, D_{2}(r, \varepsilon) \neq \emptyset$, for $\varepsilon>0$ small enough.

(d) We say that $\rho$ satisfies (UUC2) if for every $s \geqslant 0$ and $\varepsilon>0$, there exists $\eta_{2}(s, \varepsilon)>0$ depending on $s$ and $\varepsilon$ such that

$$
\delta_{2}(r, \varepsilon)>\eta_{2}(s, \varepsilon)>0, \quad \text { for } r>s .
$$

(e) We say that $\rho$ is strictly convex, (SC), if for every $x, y \in X_{\rho}$ such that $\rho(x)=\rho(y)$ and

$$
\rho\left(\frac{x+y}{2}\right)=\frac{\rho(x)+\rho(y)}{2}
$$

we have $x=y$.

Note that in Orlicz spaces, as described in Example 2.3, if the modular satisfies the $\Delta_{2}$-condition, then uniform convexity of the Luxemburg norm is equivalent to (UC1) $[1,4,8,15,16]$. But in the absence of the $\Delta_{2}$-condition, we may still have (UC1) provided the Orlicz function is uniformly convex like $\varphi_{1}(\mathrm{t})=e^{|\mathrm{t}|}-|\mathrm{t}|-1$ and $\varphi_{2}(\mathrm{t})=\mathrm{e}^{\mathrm{t}^{2}}-1[4,11,16]$.

Let us observe that for $i=1,2$, we have $\delta_{i}(r, 0)=0$, and $\delta_{i}(r, \varepsilon)$ is an increasing function of $\varepsilon$ for every fixed $r$. The following properties follow easily from Definition 3.1.

Proposition 3.2 ([10]). The following conditions characterize relationship between the above defined notions:

(a) (UUCi) implies (UCi) for $i=1,2$;

(b) $\delta_{1}(r, \varepsilon) \leqslant \delta_{2}(r, \varepsilon)$;

(c) (UC1) implies (UC2);

(d) (UC2) implies (SC);

(e) (UUC1) implies (UUC2).

Note that if $\rho$ satisfies the $\Delta_{2}$-condition, then (UC1) and (UC2) are equivalent. In the next example, we discuss these properties in the modular vector space introduced by Orlicz and studied by many authors.

Example $3.3([19,21,24])$. Consider the function $\rho$ defined on $X=\mathbb{R}^{\mathbb{N}}$ by

$$
\rho(x)=\rho\left(\left(x_{n}\right)\right)=\sum_{n=1}^{\infty}\left|x_{n}\right|^{n+1} .
$$

It is easy to check that $\rho$ is a convex modular according to Definition 2.1. Note that $\rho$ does not satisfy the $\Delta_{2}$-condition since $\rho(x)<\infty$ while $\rho(2 x)=\infty$ where $x=\left(x_{n}\right)$ with $x_{n}=1 / 2$ for any $n \geqslant 1$. Moreover, the normed vector space $\left(X_{\rho},\|\cdot\|_{\rho}\right)$ is a reflexive Banach space [19]. Using the inequality

$$
|a+b|^{p}+|a-b|^{p} \leqslant 2^{p-1}\left(|a|^{p}+|b|^{p}\right)
$$


we get

$$
\left|\frac{a+b}{2}\right|^{p}+\left|\frac{a-b}{2}\right|^{p} \leqslant \frac{1}{2}\left(|a|^{p}+|b|^{p}\right)
$$

for any $a, b \in \mathbb{R}$ and $p \geqslant 2$. This easily implies the following inequality

$$
\rho\left(\frac{x+y}{2}\right)+\rho\left(\frac{x-y}{2}\right) \leqslant \frac{1}{2}(\rho(x)+\rho(x))
$$

for any $x, y \in X_{\rho}$. Hence $\rho$ is (UC2) with $\delta_{2}(r, \varepsilon) \geqslant \varepsilon$, for any $r>0$ and $\varepsilon>0$. In fact $\rho$ is (UUC2). On the other hand, $\rho$ fails to be (UC1). Indeed, set $e_{m}=\left(x_{n}\right)$, with $x_{n}=0$ if $n \neq m$ and $x_{m}=1$, for any $m \geqslant 1$. Next, we consider the vectors

$$
x_{m}=\left(1+\frac{1}{m+1}\right) e_{m}+b e_{m+1}, \quad y_{m}=\left(1+\frac{1}{m+1}\right) e_{m}-b e_{m+1}
$$

for $m \geqslant 1$ and $1 / 2<b<1$. Hence

$$
\rho\left(x_{m}\right)=\rho\left(y_{m}\right)=\left(1+\frac{1}{m+1}\right)^{m+1}+b^{m+2}, \rho\left(\frac{x_{m}+y_{m}}{2}\right)=\left(1+\frac{1}{m+1}\right)^{m+1},
$$

and $\rho\left(x_{m}-y_{m}\right)=\rho\left(2 b e_{m+1}\right)=(2 b)^{m+2}$, which implies

$$
\lim _{m \rightarrow \infty} \rho\left(x_{m}\right)=\lim _{m \rightarrow \infty} \rho\left(y_{m}\right)=\lim _{m \rightarrow \infty} \rho\left(\frac{x_{m}+y_{m}}{2}\right)=e,
$$

and $\lim _{m \rightarrow \infty} \rho\left(x_{m}-y_{m}\right)=\infty$. This is not possible if $\rho$ is (UC1).

The above example explains why we had to introduce the two uniform convexities of the modular. In fact, almost all the papers published on the subject focus mainly on (UC1). This is important because (UC2) allows us to prove modular geometric properties which are otherwise unknown in the absence of (UC1).

The following technical lemma will be useful throughout.

Lemma 3.4. Let $\rho$ be a convex modular defined in $\mathrm{X}$ which satisfies the Fatou property. Assume $\mathrm{X}_{\rho}$ is complete and $\rho$ is (UUC2). The following properties hold.

(i) Let $\mathrm{C}$ be a nonempty $\rho$-closed convex subset of $\mathrm{X}_{\rho}$. Let $\mathrm{x} \in \mathrm{X}_{\rho}$ be such that

$$
d_{\rho}(x, C)=\inf \{\rho(x-y): y \in C\}<\infty .
$$

Then there exists a unique $c \in C$ such that $\mathrm{d}_{\rho}(x, \mathrm{C})=\rho(x-c)$.

(ii) $X_{\rho}$ satisfies the property $(R)$, i.e., for any decreasing sequence $\left\{C_{n}\right\}_{n} \geqslant 1$ of $\rho$-closed convex nonempty subsets of $X_{\rho}$ such that $\sup _{n \geqslant 1} d_{\rho}\left(x, C_{n}\right)<\infty$, for some $x \in X_{\rho}$, then we have $\bigcap_{n \geqslant 1} C_{n}$ is nonempty.

Proof. In order to prove (i), we may assume that $x \notin C$ since $C$ is $\rho$-closed. Therefore, we have $d_{\rho}(x, C)>0$. Set $R=d_{\rho}(x, C)$. Hence for any $n \geqslant 1$, there exists $y_{n} \in C$ such that $\rho\left(x-y_{n}\right)<R(1+1 / n)$. We claim that $\left\{y_{n} / 2\right\}$ is $\rho$-Cauchy. Assume otherwise that $\left\{y_{n} / 2\right\}$ is not $\rho$-Cauchy. Then there exists a subsequence $\left\{y_{\varphi(n)}\right\}$ and $\varepsilon_{0}>0$ such that $\rho\left(\left(y_{\varphi(n)}-y_{\varphi(m)}\right) / 2\right) \geqslant \varepsilon_{0}$, for any $n>m \geqslant 1$. Since $R(1+1 / n)>R / 2=s$, for any $n \geqslant 1$, we conclude that

$$
\delta_{2}\left(R(1+1 / n), 2 \varepsilon_{0} / R\right) \geqslant \eta_{2}\left(R / 2,2 \varepsilon_{0} / R\right)>0,
$$


for any $n \geqslant 1$. Since $\max \left(\rho\left(x-y_{\varphi(n)}\right), \rho\left(x-y_{\varphi(m)}\right)\right) \leqslant R(1+1 / \varphi(m))$ and

$$
\rho\left(\frac{y_{\varphi(n)}-y_{\varphi(m)}}{2}\right) \geqslant \varepsilon_{0} \geqslant R\left(1+\frac{1}{\varphi(m)}\right) \frac{\varepsilon_{0}}{2 R}
$$

for any $n>m \geqslant 1$, we conclude that

$$
\rho\left(x-\frac{y_{\varphi(n)}+y_{\varphi(m)}}{2}\right) \leqslant R\left(1+\frac{1}{\varphi(m)}\right)\left(1-\eta_{2}\left(R / 2,2 \varepsilon_{0} / R\right)\right) .
$$

Hence

$$
R=d_{\rho}(x, C) \leqslant R\left(1+\frac{1}{\varphi(m)}\right)\left(1-\eta_{2}\left(R / 2,2 \varepsilon_{0} / R\right)\right)
$$

for any $m \geqslant 1$. If we let $m \rightarrow \infty$, we get $R \leqslant R\left(1-\eta_{2}\left(R / 2,2 \varepsilon_{0} / R\right)\right)$ which is a contradiction with the facts $R>0$ and $\eta\left(R / 2,2 \varepsilon_{0} / R\right)>0$. Therefore, $\left\{y_{n} / 2\right\}$ is $\rho$-Cauchy. Since $X_{\rho}$ is $\rho$-complete, then $\left\{y_{n} / 2\right\}$ $\rho$-converges to some $y$. We claim that $2 y \in C$. Indeed, for any $m \geqslant 1$, the sequence $\left\{\left(y_{n}+y_{m}\right) / 2\right\}$ $\rho$-converges to $y+y_{m} / 2$. Since $C$ is $\rho$-closed and convex, we get $y+y_{m} / 2 \in C$. Finally the sequence $\left\{y+y_{m} / 2\right\} \rho$-converges to $2 y$, which implies $2 y \in C$. Set $c=2 y$. Since $\rho$ satisfies the Fatou property, we have

$$
\begin{aligned}
d_{\rho}(x, C) & \leqslant \rho(x-c) \\
& \leqslant \liminf _{m \rightarrow \infty} \rho\left(x-\left(y+y_{m} / 2\right)\right) \\
& \leqslant \liminf _{m \rightarrow \infty} \liminf _{n \rightarrow \infty} \rho\left(x-\left(y_{n}+y_{m} / 2\right)\right) \\
& \leqslant \liminf _{m \rightarrow \infty} \liminf _{n \rightarrow \infty}\left(\rho\left(x-y_{n}\right)+\rho\left(x-y_{m}\right)\right) / 2 \\
& =R=d_{\rho}(x, C) .
\end{aligned}
$$

Hence $\rho(x-c)=d_{\rho}(x, C)$. The uniqueness of the point $c$ follows from the fact that $\rho$ is (SC).

For the proof of (ii), we assume that $x \notin C_{n_{0}}$ for some $n_{0} \geqslant 1$. In fact, the sequence $\left\{d_{\rho}\left(x, C_{n}\right)\right\}$ is increasing and bounded. Set $\lim _{n \rightarrow \infty} d_{\rho}\left(x, C_{n}\right)=R$. We may assume $R>0$. Otherwise $x \in C_{n}$, for any $n \geqslant 1$. From (i), there exists a unique $y_{n} \in C_{n}$ such that $d_{\rho}\left(x, C_{n}\right)=\rho\left(x-y_{n}\right)$, for any $n \geqslant 1$. A similar proof will show that $\left\{y_{n} / 2\right\} \rho$-converges to some $y \in X_{\rho}$. Since $\left\{C_{n}\right\}$ are decreasing, convex and $\rho$-closed, we conclude that $2 y \in \bigcap_{n \geqslant 1} C_{n}$.

It is natural to wonder whether the property $(R)$ extends to any family of decreasing subsets.

Proposition 3.5. Let $\rho$ be a convex modular defined in X. Assume $\mathrm{X}_{\rho}$ is complete and $\rho$ is (UUC2). Let C be $\rho$-closed nonempty convex subsets of $\mathrm{X}_{\rho}$ which is $\rho$-bounded. Let $\left\{\mathrm{C}_{i}\right\}_{i \in \mathrm{I}}$ be a family of $\rho$-closed nonempty convex subsets of $C$ such that $\bigcap_{i \in F} C_{i} \neq \emptyset$, for any finite subset $F$ of $I$. Then $\bigcap_{i \in I} C_{i} \neq \emptyset$.

Proof. Let $x \in C$. Then $\sup _{i \in I} d_{\rho}\left(x, C_{i}\right) \leqslant \delta_{\rho}(C)<\infty$ holds. For any subset $F \subset I$, set $d_{F}=d_{\rho}\left(x, \bigcap_{i \in F} C_{i}\right)$. Note that if $F_{1} \subset F_{2} \subset I$ are finite subsets, then $d_{F_{1}} \leqslant d_{F_{2}}$ holds. Set

$$
d_{I}=\sup \left\{d_{\rho}\left(x, \bigcap_{i \in J} C_{i}\right), J \subset I \text { such that } \bigcap_{i \in J} C_{i} \neq \emptyset\right\} .
$$

For any $n \geqslant 1$, there exists a subset $F_{n} \subset I$ such that $d_{I}-1 / n<d_{F_{n}} \leqslant d_{I}$. Set $F_{n}^{*}=F_{1} \cup \cdots \cup F_{n}$, for $n \geqslant 1$. Then $\left\{\bigcap_{i \in F_{n}^{*}} C_{i}\right\}$ is a decreasing sequence of nonempty $\rho$-closed convex subsets of $X_{\rho}$. The 
property (R) implies $\bigcap_{i \in J} C_{i} \neq \emptyset$, where $J=\bigcup_{n \geqslant 1} F_{n}^{*}=\bigcup_{n \geqslant 1} F_{n}$. Set $K=\bigcap_{i \in J} C_{i}$. Note that $d_{\rho}(x, K)=d_{I}$ because $d_{I}-1 / n<d_{F_{n}} \leqslant d_{\rho}(x, K) \leqslant d_{I}$, for any $n \geqslant 1$. Lemma 3.4 implies the existence of a unique $y \in K$ such that $\rho(x-y)=d_{\rho}(x, K)=d_{I}$. Let $i_{0} \in I$, then

$$
\mathrm{K} \cap \mathrm{C}_{\mathrm{i}_{0}}=\bigcap_{i \in J \cup\left\{\mathfrak{i}_{0}\right\}} \mathrm{C}_{\boldsymbol{i}} \neq \emptyset,
$$

because of the same argument using the property $(R)$. Hence $d_{\rho}(x, K) \leqslant d_{\rho}\left(x, K \cap C_{i_{0}}\right) \leqslant d_{I}$. Hence $d_{\rho}\left(x, K \cap C_{i_{0}}\right)=d_{\rho}(x, K)=d_{I}$ which implies $y \in K \cap C_{i_{0}}$. Therefore, we have $y \in \bigcap_{i \in I} C_{i}$ which proves our claim.

The concept of $\rho$-type functions will play a major role in the next section.

Definition 3.6. Let $\left\{x_{n}\right\}$ be a sequence in $X_{\rho}$. Let $C$ be a nonempty subset of $X_{\rho}$. The function $\tau: C \rightarrow[0, \infty]$ defined by

$$
\tau(x)=\limsup _{n \rightarrow \infty} \rho\left(x-x_{n}\right),
$$

is called a $\rho$-type function. A sequence $\left\{c_{n}\right\}$ in $C$ is called a minimizing sequence of $\tau$ if $\lim _{n \rightarrow \infty} \tau\left(c_{n}\right)=$ $\inf _{x \in C} \tau(x)$.

The $\rho$-type functions enjoy some interesting and powerful properties.

Proposition 3.7. Assume that $\mathrm{X}_{\rho}$ is $\rho$-complete and $\rho$ satisfies the Fatou property. Let $\mathrm{C}$ be a nonempty convex and $\rho$-closed subset of $X_{\rho}$. Consider the $\rho$-type function $\tau: C \rightarrow[0, \infty]$ generated by a sequence $\left\{x_{n}\right\}$ in $X_{\rho}$. Assume $\tau_{0}=\inf _{x \in C} \tau(x)<\infty$.

(i) If $\rho$ is (UUC1), then all minimizing sequences of $\tau$ are $\rho$-convergent to the same limit.

(ii) If $\rho$ is (UUC2) and $\left\{c_{n}\right\}$ is a minimizing sequence of $\tau$, then $\left\{c_{n} / 2\right\} \rho$-converges to a point which is independent of the minimizing sequence $\left\{\mathrm{c}_{\mathrm{n}}\right\}$.

Proof. First, we assume that $\tau_{0}>0$. Let $\left\{c_{n}\right\}$ be a minimizing sequence of $\tau$. We will prove (i) and omit the proof to (ii) since it is quite similar. Assume that $\left\{c_{n}\right\}$ is not $\rho$-Cauchy. Then there exist a subsequence $\left\{\mathbf{c}_{\varphi(n)}\right\}$ of $\left\{\mathbf{c}_{n}\right\}$ and $\varepsilon_{0}>0$ such that

$$
\rho\left(\mathrm{c}_{\varphi(\mathrm{n})}-\mathrm{c}_{\varphi(m)}\right) \geqslant \varepsilon_{0}, \text { for } \mathrm{n} \neq \mathrm{m} .
$$

Fix $\varepsilon \in(0,1)$. Then there exists $n_{0} \geqslant 1$ such that for any $n \geqslant n_{0}$, we have $\tau\left(c_{\varphi(n)}\right) \leqslant \tau_{0}+\varepsilon$. For any $n>m \geqslant n_{0}$, there exists $k_{n, m} \geqslant 1$ such that for any $k \geqslant k_{n, m}$, we have

$$
\max \left(\rho\left(\mathrm{c}_{\varphi(\mathrm{n})}-\mathrm{x}_{\mathrm{k}}\right), \rho\left(\mathrm{c}_{\varphi(\mathrm{m})}-\mathrm{x}_{\mathrm{k}}\right)\right) \leqslant \tau_{0}+2 \varepsilon .
$$

Since $\rho$ is (UUC1) and

$$
\rho\left(\mathfrak{c}_{\varphi(\mathfrak{n})}-\mathbf{c}_{\varphi(m)}\right) \geqslant \varepsilon_{0} \geqslant\left(\tau_{0}+2 \varepsilon\right) \frac{\varepsilon_{0}}{\tau_{0}+2},
$$

we get

$$
\rho\left(\frac{\mathbf{c}_{\varphi(\mathfrak{n})}+\mathrm{c}_{\varphi(\mathrm{m})}}{2}-\mathrm{x}_{\mathrm{k}}\right) \leqslant\left(\tau_{0}+2 \varepsilon\right)\left(1-\eta_{2}\left(\tau_{0}, \frac{\varepsilon_{0}}{\tau_{0}+2}\right)\right)
$$

for any $k \geqslant k_{n, m}$. Hence

$$
\tau\left(\frac{\mathbf{c}_{\varphi(\mathfrak{n})}+\mathrm{c}_{\varphi(\mathrm{m})}}{2}\right) \leqslant\left(\tau_{0}+2 \varepsilon\right)\left(1-\eta_{2}\left(\tau_{0}, \frac{\varepsilon_{0}}{\tau_{0}+2}\right)\right)
$$


for any $n>m \geqslant n_{0}$, which implies

$$
\tau_{0} \leqslant\left(\tau_{0}+2 \varepsilon\right)\left(1-\eta_{2}\left(\tau_{0}, \frac{\varepsilon_{0}}{\tau_{0}+2}\right)\right) .
$$

If we let $\varepsilon \rightarrow 0$, we get

$$
\tau_{0} \leqslant \tau_{0}\left(1-\eta_{2}\left(\tau_{0}, \frac{\varepsilon_{0}}{\tau_{0}+2}\right)\right),
$$

which contradicts the fact $\tau_{0}>0$. Therefore $\left\{c_{n}\right\}$ is $\rho$-Cauchy. Since $X_{\rho}$ is $\rho$-complete, we conclude that $\left\{c_{n}\right\}$ is $\rho$-convergent. Next we show that the $\rho$-limit is independent of the minimizing sequence. Let $\left\{c_{n}^{*}\right\}$ be another minimizing sequence of $\tau$ in $C$. Define $\left\{\bar{c}_{n}\right\}$ by $\bar{c}_{2 n}=c_{n}$ and $\bar{c}_{2 n+1}=c_{n}^{*}$, for any $n \geqslant 1$. Then $\left\{\bar{c}_{n}\right\}$ is also a minimizing sequence of $\tau$ in C. Hence $\left\{\bar{c}_{n}\right\}$ is $\rho$-convergent. This fact will force both $\left\{c_{n}\right\}$ and $\left\{c_{n}^{*}\right\}$ to have the same $\rho$-limit. In order to finish the proof of Proposition 3.7, let us take care of the case $\tau_{0}=0$. For (ii), the proof is easy. Indeed, let $\left\{c_{n}\right\}$ be a minimizing sequence of $\tau$ in $C$. Then we have

$$
\rho\left(\frac{c_{n}-c_{m}}{2}\right) \leqslant \frac{1}{2} \rho\left(c_{n}-x_{k}\right)+\frac{1}{2} \rho\left(c_{m}-x_{k}\right)
$$

for any $n, m, k \geqslant 1$. Hence

$$
\tau\left(\frac{c_{n}-c_{m}}{2}\right) \leqslant \frac{1}{2} \tau\left(c_{n}\right)+\frac{1}{2} \tau\left(c_{m}\right)
$$

for any $n, m \geqslant 1$. Since $\lim _{n \rightarrow \infty} \tau\left(c_{n}\right)=\tau_{0}=0$, we conclude that $\left\{c_{n} / 2\right\}$ is $\rho$-Cauchy. Since $X_{\rho}$ is $\rho$-complete, we conclude that $\left\{c_{n} / 2\right\}$ is $\rho$-convergent. The same idea used before will show that the $\rho$-limit is in fact independent of the minimizing sequence. The proof of (ii) in this case is little bit complicated. For any $n \geqslant 1$, consider $K_{n}$ to be the intersection of all $\rho$-closed convex subsets of $X \rho$ which contains $x_{i}$, for $i \geqslant n$. Clearly $\left\{K_{n}\right\}$ are decreasing. Let $x \in C$ such that $\tau(x)<\infty$. For any $\varepsilon>0$, there exists $k_{0} \geqslant 1$ such that

$$
\rho\left(x-x_{k}\right) \leqslant \tau(x)+\varepsilon, \text { for } k \geqslant k_{0} .
$$

Hence $x_{k} \in B_{\rho}(x, \tau(x)+\varepsilon)$, for any $k \geqslant k_{0}$. Since $\rho$ satisfies the Fatou property, $B_{\rho}(x, \tau(x)+\varepsilon)$ is $\rho$-closed. Hence $K_{n} \subset B_{\rho}(x, \tau(x)+\varepsilon)$, for any $n \geqslant k_{0}$, which implies

$$
d_{\rho}\left(x, K_{n}\right)=\inf \left\{\rho(x-y): y \in K_{n}\right\} \leqslant \tau(x)+\varepsilon
$$

for any $n \geqslant k_{0}$. Using the property (R) satisfied by $X_{\rho}$, we get $K=\bigcap_{n \geqslant k_{0}} K_{n}$ is not empty. Since $\left\{K_{n}\right\}$ are decreasing, we get $K=\bigcap_{n \geqslant 1} K_{n}$. Let $z \in K$. Then $z \in K_{n}$, for any $n \geqslant k_{0}$. Hence $z \in B_{\rho}(x, \tau(x)+\varepsilon)$ which implies $\rho(x-z) \leqslant \tau(x)+\varepsilon$. Since $\varepsilon$ was taken arbitrarily, we get $\rho(x-z) \leqslant \tau(x)$. Let $\left\{c_{n}\right\}$ be a minimizing sequence of $\tau$ in $C$. Since $\lim _{n \rightarrow \infty} \tau\left(c_{n}\right)=\tau_{0}=0$ and $\rho\left(c_{n}-z\right) \leqslant \tau\left(c_{n}\right)$, for any $n \geqslant 1$, we conclude that $\left\{c_{n}\right\} \rho$-converges to $z$. Hence $z \in C$ and is independent of the minimizing sequence. This completes the proof of Proposition 3.7.

In the next section, we discuss some applications of the ideas discussed above to the fixed point theory of mappings which are Lipschitzian in the modular sense.

\section{Some fixed point results}

In this section, we initiate the analogue to the fundamental metric fixed point results in modular vector spaces. Throughout $X$ is a vector space and $\rho$ a convex modular function. Let us start with the modular definitions in the modular sense of Lipschitzian mappings. 
Definition 4.1. Let $\rho$ be a modular defined on a vector space $X$. Let $C \subset X_{\rho}$ be nonempty. A mapping $\mathrm{T}: \mathrm{C} \rightarrow \mathrm{C}$ is called $\rho$-Lipschitzian if there exists a constant $\mathrm{K} \geqslant 0$ such that

$$
\rho(T(x)-T(y)) \leqslant K \rho(x-y), \quad \forall x, y \in C .
$$

If $\mathrm{K}<1$, then $\mathrm{T}$ is called $\rho$-contraction. And if $\mathrm{K}=1, \mathrm{~T}$ is called $\rho$-nonexpansive. A point $x \in \mathrm{C}$ is called a fixed point of $T$ if $T(x)=x$. The set of fixed points of $T$ will be denoted by $\operatorname{Fix}(T)$.

The first result is the modular version of the Banach Contraction Principle.

Theorem 4.2. Assume $\mathrm{X}_{\rho}$ is $\rho$-complete. Let $\mathrm{C}$ be a nonempty $\rho$-closed subset of $\mathrm{X}_{\rho}$. Let $\mathrm{T}: \mathrm{C} \rightarrow \mathrm{C}$ be a $\rho$ contraction mapping. Then $\mathrm{T}$ has a fixed point $z$ if and only if $\mathrm{T}$ has a $\rho$-bounded orbit. Moreover if $\rho(x-z)<\infty$, then $\left\{\mathrm{T}^{\mathrm{n}}(\mathrm{x})\right\}$ o-converges to $z$, for any $\mathrm{x} \in \mathrm{C}$.

Proof. It is obvious that if T has a fixed point $z$, the orbit $\left\{\mathrm{T}^{\mathrm{n}}(z)\right\}$ is $\rho$-bounded. Assume there exists $x_{0} \in \mathrm{C}$ such that $\left\{T^{\mathfrak{n}}\left(x_{0}\right)\right\}$ is $\rho$-bounded, i.e.,

$$
\delta_{\rho}\left(x_{0}\right)=\sup \left\{\rho\left(T^{n}\left(x_{0}\right)-T^{m}\left(x_{0}\right)\right) ; n, m \in \mathbb{N}\right\}<\infty .
$$

Since $T$ is a $\rho$-contraction mapping there exists $K<1$ such that

$$
\rho(T(x)-T(y)) \leqslant K \rho(x-y), \quad x, y \in C .
$$

Hence

$$
\rho\left(T^{n}\left(x_{0}\right)-T^{n+h}\left(x_{0}\right)\right) \leqslant K^{n} \rho\left(x_{0}-T^{h}\left(x_{0}\right)\right) \leqslant K^{n} \delta_{\rho}\left(x_{0}\right)
$$

for any $n, h \in \mathbb{N}$. Since $K<1$, we conclude that $\left\{T^{n}\left(x_{0}\right)\right\}$ is $\rho$-Cauchy. Since $X_{\rho}$ is $\rho$-complete, $\left\{T^{n}\left(x_{0}\right)\right\}$ $\rho$-converges to some $z \in X_{\rho}$. Since $C$ is $\rho$-closed, we obtain that $z \in C$. Let us prove that $z$ is in fact a fixed point of $T$. Indeed we have

$$
\rho\left(T^{n+1}\left(x_{0}\right)-T(z)\right) \leqslant K \rho\left(T^{n}\left(x_{0}\right)-z\right)
$$

for any $n \in \mathbb{N}$. Hence

$$
\begin{aligned}
\rho\left(\frac{z-\mathrm{T}(z)}{2}\right) & =\rho\left(\frac{z-\mathrm{T}^{\mathrm{n}+1}\left(\mathrm{x}_{0}\right)}{2}+\frac{\mathrm{T}^{\mathrm{n}+1}\left(\mathrm{x}_{0}\right)-\mathrm{T}(z)}{2}\right) \\
& \leqslant \frac{1}{2} \rho\left(z-\mathrm{T}^{\mathrm{n}+1}\left(\mathrm{x}_{0}\right)\right)+\frac{\mathrm{K}}{2} \rho\left(\mathrm{T}^{\mathrm{n}}\left(\mathrm{x}_{0}\right)-z\right)
\end{aligned}
$$

for any $n \in \mathbb{N}$. If we let $n \rightarrow \infty$, we obtain

$$
\rho\left(\frac{z-\mathrm{T}(z)}{2}\right)=0
$$

which implies that $T(z)=z$. Let $x \in C$ be such that $\rho(x-z)<\infty$. Then we have

$$
\rho\left(T^{n}(x)-z\right)=\rho\left(T^{n}(x)-T^{n}(z)\right) \leqslant K^{n} \rho(x-z)
$$

for any $n \in \mathbb{N}$. Since $K<1$, we conclude that $\left\{T^{n}(x)\right\} \rho$-converges to $z$.

Remark 4.3. One may wonder what happened to the uniqueness of the fixed point in the fundamental Banach Contraction Principle. Since $\rho$ is allowed to take infinite values, this conclusion may fail. But Theorem 4.2 allows us to conclude that if $z_{1}$ and $z_{2}$ are two fixed points of T such that $\rho\left(z_{1}-z_{2}\right)<\infty$, then we have $z_{1}=z_{2}$. In particular, if $C$ is $\rho$-bounded, then $T$ has a unique fixed point in $C$.

Next we investigate the case of $\rho$-nonexpansive mappings. First note that if $C$ is convex, then fix $x_{0} \in C$ and $\varepsilon \in(0,1)$ and define $T_{\varepsilon}: C \rightarrow C$ by

$$
\mathrm{T}_{\varepsilon}(x)=\varepsilon x_{0}+(1-\varepsilon) \mathrm{T}(x) .
$$

Since $\rho$ is convex, we deduce that $T_{\varepsilon}$ is a $\rho$-contraction. Assume that $C$ is $\rho$-bounded $\rho$-closed and $X_{\rho}$ is 
$\rho$-complete, then $T_{\varepsilon}$ has a unique fixed point $x_{\varepsilon} \in C$. Hence we have

$$
\varepsilon x_{0}+(1-\varepsilon) \mathrm{T}\left(x_{\varepsilon}\right)=x_{\varepsilon},
$$

which implies

$$
\rho\left(x_{\varepsilon}-T\left(x_{\varepsilon}\right)\right)=\rho\left(\varepsilon\left(x_{0}-T\left(x_{\varepsilon}\right)\right) \leqslant \varepsilon \rho\left(x_{0}-T\left(x_{\varepsilon}\right) \leqslant \varepsilon \delta_{\rho}(C) .\right.\right.
$$

Since $\varepsilon$ was chosen arbitrarily in $(0,1)$, we get $\inf _{x \in C} \rho(x-T(x))=0$. This implies that we almost have a fixed point. Therefore, there exists a sequence $\left\{x_{n}\right\}$ in $C$ such that $\lim _{n \rightarrow \infty} \rho\left(x_{n}-T\left(x_{n}\right)\right)=0$. Such sequence will be called a $\rho$-almost fixed point sequence of $T$. Assume that $C$ is $\rho$-compact, then we may assume that $T$ has a $\rho$-almost fixed point sequence $\left\{x_{n}\right\}$ which is $\rho$-convergent to some $z \in C$. Hence

$$
\rho\left(\frac{z-T(z)}{3}\right) \leqslant \frac{1}{3} \rho\left(z-x_{n}\right)+\frac{1}{3} \rho\left(x_{n}-T\left(x_{n}\right)\right)+\frac{1}{3} \rho\left(T\left(x_{n}\right)-T(z)\right),
$$

which implies

$$
\rho\left(\frac{z-T(z)}{3}\right) \leqslant \frac{2}{3} \rho\left(z-x_{n}\right)+\frac{1}{3} \rho\left(x_{n}-T\left(x_{n}\right)\right)
$$

for any $n \geqslant 1$. If we let $n \rightarrow \infty$, we get

$$
\rho\left(\frac{z-\mathrm{T}(z)}{3}\right)=0
$$

which implies that $\mathrm{T}(z)=z$.

Theorem 4.4. Assume $\mathrm{X}_{\rho}$ is $\rho$-complete. Let $\mathrm{C}$ be a nonempty $\rho$-compact convex $\rho$-bounded subset of $\mathrm{X}_{\rho}$. Let $\mathrm{T}: \mathrm{C} \rightarrow \mathrm{C}$ be a $\rho$-nonexpansive mapping. Then $\mathrm{T}$ has a fixed point.

Clearly $\rho$-compactness is a strong assumption. In order to weaken this assumption and prove a similar result to Browder-Göhde fixed point theorems $[3,7]$, we need to assume the uniform convexity of the modular.

Theorem 4.5. Assume that $\mathrm{X}_{\rho}$ is $\rho$-complete and $\rho$ satisfies the Fatou property. Assume $\rho$ is (UUC1). Let $\mathrm{C}$ be a nonempty $\rho$-closed convex $\rho$-bounded subset of $\mathrm{X}_{\rho}$. Let $\mathrm{T}: \mathrm{C} \rightarrow \mathrm{C}$ be a $\rho$-nonexpansive mapping. Then $\mathrm{T}$ has a fixed point. Moreover Fix $(\mathrm{T})$ is $\rho$-closed and convex.

Proof. Let $\mathrm{x}_{0} \in \mathrm{C}$. Consider the $\rho$-type function $\tau: \mathrm{C} \rightarrow[0, \infty]$ defined by

$$
\tau(x)=\limsup _{n \rightarrow \infty} \rho\left(x-T^{n}\left(x_{0}\right)\right) .
$$

Note that since $\left\{T^{n}\left(x_{0}\right)\right\} \subset C$ and $C$ is $\rho$-bounded, we have $\tau(x) \leqslant \delta_{\rho}(C)<\infty$, for any $x \in C$. Moreover, we have

$$
\tau(T(x))=\limsup _{n \rightarrow \infty} \rho\left(T(x)-T^{n}\left(x_{0}\right)\right) \leqslant \limsup _{n \rightarrow \infty} \rho\left(x-T^{n-1}\left(x_{0}\right)\right)=\tau(x)
$$

for any $x \in C$. Let $\left\{c_{n}\right\}$ be a minimizing sequence of $\tau$ in $C$. It is clear that $\left\{T\left(c_{n}\right)\right\}$ is also a minimizing sequence of $\tau$. Using Proposition 3.7, we conclude that $\left\{c_{n}\right\}$ and $\left\{T\left(c_{n}\right)\right\} \rho$-converge to the same point $z \in$ C. Since

$$
\rho\left(T\left(c_{n}\right)-T(z)\right) \leqslant \rho\left(c_{n}-z\right)
$$

for $n \geqslant 1$, we conclude that $\left\{T\left(c_{n}\right)\right\}$ also $\rho$-converges to $T(z)$. The uniqueness of the $\rho$-limit implies that $\mathrm{T}(z)=z$. Hence $\operatorname{Fix}(T)$ is not empty. Let us prove it is $\rho$-closed and convex. Let $\left\{x_{n}\right\}$ be in Fix $(T)$ which $\rho$-converges to some $x \in \mathrm{C}$. We have

$$
\rho\left(x_{n}-T(x)\right)=\rho\left(T\left(x_{n}\right)-T(x)\right) \leqslant \rho\left(x_{n}-x\right), \quad n \geqslant 1 .
$$


This will imply that $\left\{x_{n}\right\}$ also $\rho$-converges to $T(x)$. The uniqueness of the $\rho$-limit implies that $x=T(x)$, i.e., $x \in \operatorname{Fix}(T)$. Hence $\operatorname{Fix}(T)$ is $\rho$-closed. Let us finish the proof of Theorem 4.5 by showing that $\operatorname{Fix}(T)$ is convex. Let $x, y \in \operatorname{Fix}(T)$. Assume that $x \neq y$. Let us prove that $z=\frac{x+y}{2} \in \operatorname{Fix}(T)$. Set $u=x-\frac{z+T(z)}{2}$ and $v=\frac{z+\mathrm{T}(z)}{2}-y$. Then we have

$$
\rho(u) \leqslant \rho\left(\frac{x-y}{2}\right), \quad \rho(v) \leqslant \rho\left(\frac{x-y}{2}\right), \quad \rho\left(\frac{u+v}{2}\right)=\rho\left(\frac{x-y}{2}\right) .
$$

Since $\rho$ is (UUC2), it is (SC). Hence $u=v$ which implies $T(z)=z$, i.e., $z \in \operatorname{Fix}(T)$.

As an application to Theorem 4.5, we have the following common fixed point.

Theorem 4.6. Assume that $\mathrm{X}_{\rho}$ is $\rho$-complete and $\rho$ satisfies the Fatou property. Assume $\rho$ is (UUC1). Let $\mathrm{C}$ be a nonempty $\rho$-closed convex $\rho$-bounded subset of $\mathrm{X}_{\rho}$. Let $\mathrm{T}_{1}, \mathrm{~T}_{2}, \cdots, \mathrm{T}_{\mathrm{n}}: \mathrm{C} \rightarrow \mathrm{C}$ be a finite family of $\rho$-nonexpansive mappings which are commutative. Then $\left\{T_{i}\right\}_{1 \leqslant i \leqslant n}$ have a common fixed point. Moreover $\bigcap_{i=1}^{n} \operatorname{Fix}\left(T_{i}\right)$ is $\rho$-closed and convex.

Proof. It is enough to prove the conclusion for $n=2$. Since $T_{1}$ and $T_{2}$ are commutative, then we have $T_{2}\left(\operatorname{Fix}\left(T_{1}\right)\right) \subset \operatorname{Fix}\left(T_{1}\right)$. The restriction of $T_{2}$ to $\operatorname{Fix}\left(T_{1}\right)$ has a fixed point by Theorem 4.5. Since both Fix $\left(T_{1}\right)$ and $\operatorname{Fix}\left(T_{2}\right)$ are $\rho$-closed and convex, then $\operatorname{Fix}\left(T_{1}\right) \cap \operatorname{Fix}\left(T_{2}\right)$ is a nonempty $\rho$-closed and convex subset of $X_{\rho}$.

It is then natural to ask whether the conclusion of Theorem 4.6 is still valid for any commutative family of $\rho$-nonexpansive mappings. A direct implication of Proposition 3.5 and Theorem 4.6 is the following result.

Theorem 4.7. Assume that $\mathrm{X}_{\rho}$ is $\rho$-complete and $\rho$ satisfies the Fatou property. Assume $\rho$ is (UUC1). Let $\mathrm{C}$ be a nonempty $\rho$-closed convex $\rho$-bounded subset of $\mathrm{X}_{\rho}$. Let $\mathrm{T}_{i}: \mathrm{C} \rightarrow \mathrm{C}$, for $\mathrm{i} \in \mathrm{I}$, be a family of $\rho$-nonexpansive mappings which are commutative. Then $\left\{\mathrm{T}_{i}\right\}_{i \in \mathrm{I}}$ have a common fixed point. Moreover $\bigcap_{i \in \mathrm{I}} \operatorname{Fix}\left(\mathrm{T}_{i}\right)$ is $\rho$-closed and convex.

\section{Acknowledgment}

This work was supported by the Deanship of Scientific Research (DSR), King Abdulaziz University, Jeddah, under Grant No. (D-057-363-1438). The authors, therefore, gratefully acknowledge the DSR technical and financial support.

\section{References}

[1] V. A. Akimovič, The uniform convexity and uniform smoothness of Orlicz spaces, (Russian) Teor. Funkciǔ Funkcional. Anal. i Priložen., 15 (1972), 114-221. 3

[2] Z. Birnbaum, W. F. Orlicz, Über die Verallgemeinerung des Begriffes der zueinander konjugierten Potenzen, Studia Math., 3 (1931), 1-67. 2.3

[3] F. E. Browder, Nonexpansive nonlinear operators in a Banach space, Proc. Nat. Acad. Sci. U.S.A., 54 (1965), $1041-1044$. 4

[4] S.-T. Chen, Geometry of Orlicz spaces, With a preface by Julian Musielak, Dissertationes Math. (Rozprawy Mat.), 356 (1996), 204 pages. 3

[5] L. Diening, P. Harjulehto, P. Hästö, M. Ruẑiĉka, Lebesgue and Sobolev spaces with variable exponents, Lecture Notes in Mathematics, Springer, Heidelberg, (2011). 1, 3

[6] T. Dominguez-Benavides, M. A. Khamsi, S. Samadi, Asymptotically regular mappings in modular function spaces, Sci. Math. Jpn., 53 (2001), 295-304. 2.5

[7] D. Göhde, Zum Prinzip der kontraktiven Abbildung, (German) Math. Nachr., 30 (1965), 251-258. 4 
[8] A. Kamińska, On uniform convexity of Orlicz spaces, Nederl. Akad. Wetensch. Indag. Math., 44 (1982), 27-36. 3

[9] M. A. Khamsi, W. A. Kirk, An introduction to metric spaces and fixed point theory, Pure and Applied Mathematics (New York), Wiley-Interscience, New York, (2001). 1

[10] M. A. Khamsi, W. M. Kozlowski, Fixed point theory in modular function spaces, With a foreword by W. A. Kirk. Birkhäuser/Springer, Cham, (2015). 1, 2, 3.1, 3.2

[11] M. A. Khamsi, W. M. Kozlowski, S.-T. Chen, Some geometrical properties and fixed point theorems in Orlicz spaces, J. Math. Anal. Appl., 155 (1991), 393-412. 3

[12] M. A. Khamsi, W. K. Kozlowski, S. Reich, Fixed point theory in modular function spaces, Nonlinear Anal., 14 (1990), 935-953. 2.4

[13] O. Kováčik, J. Rákosník, On spaces $L^{p(x)}$ and $W^{k, p}(x)$, Czechoslovak Math. J., 41 (1991), 592-618. 1

[14] W. M. Kozlowski, Modular function spaces, Monographs and Textbooks in Pure and Applied Mathematics, Marcel Dekker, Inc., New York, (1988). 2

[15] W. A. J. Luxemburg, Banach function spaces, Thesis, Technische Hogeschool te Delft, (1955), 70 pages. 3

[16] H. W. Milnes, Convexity of Orlicz spaces, Pacific J. Math., 7 (1957), 1451-1486. 3

[17] J. Musielak, Orlicz spaces and modular spaces, Lecture Notes in Mathematics, Springer-Verlag, Berlin, (1983). 2.1, 2, 3

[18] H. Nakano, Modulared semi-ordered linear spaces, Maruzen Co., Ltd., Tokyo, (1950), 288 pages. 1, 2.1

[19] H. Nakano, Modulared sequence spaces, Proc. Japan Acad., 27 (1951), 508-512. 3.3

[20] H. Nakano, Topology of linear topological spaces, Maruzen Co. Ltd., Tokyo, (1951), 281 pages. 1, 3

[21] W. Orlicz, Über konjugierte exponentenfolgen, Studia Math., 3 (1931), 200-211. 1, 3.3

[22] K. Rajagopal, M. Ružička, On the modeling of electrorheological materials, Mech. Res. Commun., 23 (1996), $401-407$. 1

[23] M. Ružička, Electrorheological fluids: modeling and mathematical theory, Lecture Notes in Mathematics, SpringerVerlag, Berlin, (2000). 1

[24] K. Sundaresan, Uniform convexity of Banach spaces $1\left(\left\{p_{i}\right\}\right)$, Studia Math., 39 (1971), 227-231. 3.3 